\title{
LA NARRATIVITÉ DANS L'ANCIEN TESTAMENT COMME PROBLÈME HERMÉNEUTIQUE
}

Erich Auerbach a bien souligné la différence entre les narrations chez Homère et celles de la Bible ${ }^{1}$. Le style homérique c'est un style du ,premier plan” (vordergründig), c'est-à-dire tous les éléments du récit sont coordonnés, tandis que les récits bibliques se caractérisent par un „plan profond” (hintergründig), où les événements et les personnages se trouvent comme si sur un fond ${ }^{2}$. La constation d'Auerbach touche juste notre problématique, notamment comment interpréter les récits de l'Ancien Testament. Il suffira de jeter un coup d'oeil sur la bibliographie sur les récits biblique et leurs analyses, pour se rendre compte de l'ampleur de la question. Pendant la dernière décennie les études sur les narrations bibliques ont paru d'une manière remarquable ${ }^{3}$. On attire même l'attention sur la recherche automatisée sur lest textes narratifs de la Bible. Tout recemment on a analysé, à l'aide de l'ordinateur, la fréquence et la distribution des formules épiques dans les narrations de la Bible hebraîque.

D'entré je dois dire qu'il ne peut être question ici que d'un rapide survol du théme choisi. „Il est extrêmement difficile de parler du sens et d'en dire quelque chose du sensé" 4 . Car, à vrai dire, la question herméneutique c'est une question avant tout du sens.

${ }^{1}$ Mimesis. La représentation de la réalité dans la littérature occidentale. Trad. de l'allemand par C. Heim, Paris 1987: „J'ai nommé [...] le style homérique un style „du premier plan”, du fait qu'en dépit de maintes anticipations et de maints retours en arrière il présente toujours ce qui est en train d'être conté comme un pur présent, sans perspective. L'examen du texte élohiste nous apprend que notre terme peut être pris en un sens encore plus étendu et plus profond. Il montre que même l'individu isolé est susceptible d'être représenté comme ayant un 'arrière-plan'"' (p. 20).

2 „Les deux styles constituent, par leur antinomie, des types fondamentaux: l'un décrit les événements en les extériorisant [...], l'autre met en valeur certains élements pour en laisser d'autres dans l'ombre" (ibid., p. 33).

${ }^{3}$ Voir p.ex.: R. Alt e r, The Art of Biblical Narrative, New York 1981; A. B e rlin, Poetics and Interpretation of Biblical Narrative, Sheffield 1983; M. S t e r n be rg, The Poetics of Biblical Narrative. Ideological Literature and the Drama of Reading, Bloomington 1985; R.W. F u n k, The Poetics of Biblical Narrative, Sonoma 1988; La Narration. Quand le récit devient communication. Sous la dir. de P. B ühle r et J.-F. H a berm a ch e r, Genève 1988; en outre les indications bibliographiques chez J.-N. Ale t ti, L'art de raconter Jésus Christ, Paris 1989.

${ }^{4}$ Je répète ce qui a écrit en 1970 A. Greimas (Du sens. Essais sémiotique, Paris 1970, p. 7). 
Je tiens à expliquer le sens du mot „narrativité” qui est un néologisme ${ }^{5}$. Par une narrativité je comprends un certain mode du fonctionnement du langage en tant qu'un texte qui raconte. Au cours des dernières annés on a fait l'objet d'une élaboration conceptuelle spécialement en France (on peut citer R. Barthes, J. Derrida, Ph. Sollers, J. Kristeva) le texte qui fait de la langue un travail en remontant à ce qui la précède. Les sémioticiens sommençaient de dire sur une productivité d'un texte par l'opposition à tout usage communicatif et répresentatif, donc réproductif du langage ${ }^{6}$. Mais je dois me séparer de la narratologie, qui a été créée par les sémioticiens, avant tout français, sans attirer l'attention sur la vérité des récits. J'accepte un texte „qui travaille”, c'est-à-dire qui produit un sens (on utilisera le terme crucial „productivité”) mais à condition qu'il s'agisse d'une transmission du message; par où il faut entendre qu'il y a une liaison entre la langue d'usage, destinée à communiquer, et les structures sous-jacentes qui pointent le sens. „Travailler le texte” — si l'on adopte déjà une telle expression des sémioticiens - c'est donc explorer comment le texte travaille, c'est-à-dire constater le travail de différenciation, stratification et confrontation qui se pratique dans le récit et produit une chaîne signifiante communicative. La réconstruction et l'intégration des ces éléments seront, en fin de compte - d'après H.-G. Gadamer - une tâche de l'herméneutique 7 .

En d'autres termes, par une narrativité on comprend tous les procès narratifs qui possèdent trois protagonistes au moins: le personnage (il ou elle), le narrateur (je) et le lecteur (tu), c'est-à-dire, celui dont on parle, celui qui parle, celui à qui on parle.

Parfois l'image du narrateur est dédoublée; dès que le narrateur est présenté dans le texte, on doit accepter l'existence d'un auteur implicite au texte, on doit accepter l'existence d'un auteur implicite au texte qui organise le texte même. Une telle existence varie d'un texte à l'autre: le narrateur peut être un des personnages principaux (ce qui s'opère dans un récit à la première personne), ou bien simplement émettre un jugement de valeur pour accéder ainsi à l'existence. Quant au lecteur, très souvent l'image du narrateur et celle du lecteur coîncident, mais il ne doit pas plus être confondu avec les lecteurs réels.

Ce sont les rapports entre: auteur implicite, narrateur, personnages et lecteur implicite qui définissent, dans leur vairété, la problematique du vrai sens d'une narration. On peut distinguer plusieurs variables susceptibles de combinaison ${ }^{8}$ :

5 „Narrativité, en sémiotique, ensemble des traits caractéristiques de la narration" (Trésor de la langue française. Dictionnaire de la langue du XIXe et du XXe siècle (1789-1960), vol. XI, Gallimard, Paris 1985).

6 Voir: J. Kristeva, Semeiotiké, Paris 1969; O. Ducrot, T. Todorov, Dictionnaire encyclopédique des sciences du langage, Paris 1972, passim.

7 „Rekonstruktion und Integration als hermeneutische Aufgaben” (H.-G. G a da me r, Wahrheit und Methode, Tübingen 1975, p. 157 - 161).

${ }^{8}$ Cf. O. D u crot, T. Tod o rov, Dictionnaire (voir note 6), p. $412-416$ et une bibliographie là-bas citée. 
1) Le récit peut se présenter comme allant de soi, naturel et transparent; ou bien, au contraire, il peut se trouver représenté dans le texte.

2) Il y a un ou plusieurs narrateurs; dans de dernier cas, ceux-ci se situent ou au même niveau, ou à des niveaux différents.

3) Le narrateur est présent soit au niveau de l'univers évoqué, soit à celui du récit, soit encore à celui des personnages. Dans ce dernier cas, celui-ci peut encore être agent ou témoin.

4) Le narrateur décrit l'univers mental du personnage de l'intérieur ou de l'extérieur. Mais on peut distinguer aussi des degrès de „profondeur” du narrateur; il s'agit de divers types de connaissances. Il faut rattacher ici le phénomène évoqué dans la Poétique d'Aristote, sous le nom de reconnaissance, où, à la place de la connaissance correcte, on trouvait l'ignorance ou l'erreur.

Il existe deux types principaux de narration: récit objectif et récit subjectif. La narration est présentée soit objectivement, au nom de l'auteur, comme une simple information sans qu'on nous explique comment nous prenons connaissance de ces événements, soit subjectivement, au nom d'un narrateur, d'un certain personnage. On préfere aussi parler de l'opposition entre points de vue interne et externe.

Prenons comme exemple Nombres $11-12^{9}$. Il y a deux niveaux de description. Au premier niveau de récit c'est Yahweh qui mêne le peuple élu vers la Terre promise, Mais au second niveau c'est Moïse qui intercède comme médiateur auprès Yahweh en faveur du peuple. La narration analysée pourrait être distribuée, grâce au modèle actantiel de A. J. Greimas, de façon parallèle:

$\begin{array}{lll}\text { sujet } & - & \text { Moïse } \\ \text { objet } & - & \text { Terre promise } \\ \text { destinateur } & - & \text { Yahweh } \\ \text { destinataire } & - & \text { peuple } \\ \text { opposant } & - & \text { insubordination des personnes } \\ \text { adjuvant } & - & \text { intercéssion }\end{array}$

Mais les textes „qui travaillent” d'une manière particulière, ce sont les discours dans l'Ancien Testament qui portent une dimension narrative comme les formules de la foi et qu'on appelera les „confessions narratives” ${ }^{10}$. Dieu confessé dans ces formules de la foi se révèle d'abord comme le Dieu „raconté”: „le Dieu d'Abraham, d'Isaac et de Jacob”. C'est une histoire qui est confessée dans un récit au double sens d'un événement his torique et narrative, où l'on peut constater le travail de différenciation, stratification et l'intégration. C'est ce qui distingue la confession narrative de foi (judéo-chrétienne) de toute

\footnotetext{
${ }^{9}$ Voir: D. J o bling, The Sense of Biblical Narrative: Three Structural Analyses in the Old Testament (I Samuel 13 - 31, Numbers 11 - 12, I Kings 17-18), Sheffield 1978, chapter II.

${ }^{10} \mathrm{~L}$ 'expression est due à J.-P. S o n n e t, La parole consacrée. Théorie des actes de langage, linguistique de l'énonciation et parole de la foi, Louvain-la-Neuve 1984, p. 144.
} 
forme de mythe ou de gnose. La confession investit le récit, mais elle est aussi portée par lui; le texte laisse „se produire” ce qu'il raconte.

Pour illustrer ce qui vient d'être dit, on pourrait faire ici référence au Credo de Deutéronome 26,5 - 9 (l'expression de G. von $\mathrm{Rad}^{11}$ ) qui présente tous les signes d'une haute antiquité et qui récapitule les faits principaux de l'histoire du salut, de l'époque patriarcale jusqu'à la conquête du pays de Canaan, et cela avec une concentration extrême sur les faits historiques et objectifs. Ce récitconfession comme un noyau kérygmatique a accueilli de diverses narrations telles que les sagas des patriarches, les mythes des origines devenus récits de la création, et même aussi des éléments non narratifs comme les documents legislatifs du Lévitique ou des theologumena comme la révélation du Nom au mont Horeb. Confessé, le récit se délivre comme symbole, c'est-à-dire, il conjoigne l'événement et la parole révélante de Dieu.

Dans cette manière, on peut dégager un phénomène linguistique et littéraire à double face: na r r a tif et kéry g m a tique. Comme l'écrit P. Ricoeur: „narratifs par cette espèce d'acollement bout-àbout de traditions innombrables, et kerygmatique, par cette espèce de profession de foi centrale de la théologie de l'Alliance qui arme (donne son armature) le récit"12. C'est cette articulation du narratif et du kerygmatique qui est caractéristique de la narrativité biblique. Ce bient de la nature du mot mot hébraïque dabar. Ce mot est un des termes primitifs, dans le langage biblique, pour désigner l'histoire.

Il est à élaborer une grammaire narrative de l'Ancien Testament qui devrait d'emblée prévoir tous les valeurs modales (vouloir, savoir, pouvoir) et objectives (faire). Il faut méner l'étude analytique de l'articulation du narratif et du kérygmatique avec les moyens qu'offrent les modeles linguistiques et les analyses littéraires comparées à une herméneutique qui s'intéresse au „monde du texte", c'est-à-dire, qui s'intéresse autant aux systèmes sous-jacents dont le récit serait l'expression diachronique, qu'à la manière dont la narrativité se profère dans sa successivité. L'herméneutique traditionnelle - romantique et diltheyenne - parlait de l'éclipse des narrations bibliques ${ }^{13}$, mais une nouvelle perspective nous permettra de „se comprendre devant le texte”.

Terminons par les remargues de M. Buber ${ }^{14}$ :

Le récit est lui-même événement, il a la consécration d'une action sainte. Le récit fait plus que refléter autre chose: l'essence sainte qui est attestée en lui continue de vivre en lui. Le miracle qu'on raconte est de nouveau puissant [...] On demanda à un rabbin dont le grand-père avait été un disciple de Baalschem, de raconter une histoire. Une histoire, dit-il, on doit la raconter de telle

11 Theologie des Alten Testaments. Bd I: Die Theologie der geschichtlichen Überlieferungen Israels, München 1962, p. 135.

12 Les incidences théologiques des recherches actuelles concernant le langage (Institut d'Etudes Oecuméniques), Paris 1969, p. 63.

${ }_{13}$ Voir: H. W. Fr e i, The Eclipse of Biblical Narrative. A Study in Eighteenth and Nineteenth Century Hermeneutics, New Haven — London 1974.

14 Werke III, München 1963, p. 71. 
sorte qu'elle soit elle-même une aide. Et il raconta: Mon grand-père était paralysé. Un jour, on le pria de raconter une histoire sur son maître. Alors il raconta comment le saint Baalschem avait l'habitude, en priant, de sauter et de danser. Mon grand-père se leva et raconta, et le fait de raconter l'enleva de telle sorte qu'il dut, en sautant et en dansant, montrer comment le maître avait fait. Dès cette heure il fut guéri. C'est ainsi qu'on doit raconter des histoires.

Veuillez m'excuser que je n'ai pas pu présenter mon short paper dans cette manière! ${ }^{15}$

${ }^{15}$ C'est un abrégé du texte présenté pendant le XIIIe Congrès de l'International Organization for the Study of the Old Testament (IOSOT), à Leuven, le 30. août 1989. 